\title{
ON THE AMPLITUDE EQUATIONS \\ ARISING AT THE ONSET OF THE OSCILLATORY INSTABILITY IN PATTERN FORMATION*
}

JOSÉ M. VEGA ${ }^{\dagger}$

\begin{abstract}
A well-known system of two amplitude equations is considered that describes the weakdy nonlinear evolution of many nonequilibrium systems at the onset of the so-called oscillatory instability. Those equations depend on a small parameter, $\varepsilon$, that is a ratio between two distinguished spatial scales. In the limit $\varepsilon \rightarrow 0$, a simpler asymptotic model is obtained that consiste of two complex cubic Ginzburg-Landau equations, coupled only by spatially averaged terms.
\end{abstract}

Key words. pattern formation, oscillatory instability, amplitude equations, Ginzburg-Landau equations

AMS subject classifications. 35K55, 35B25, 35B32, 35B35

1. Introduction and formulation. This paper deals with the following parabolic problem:

$$
\begin{aligned}
& A_{t}=(1+i \alpha) A_{x x}-\varepsilon-1 A_{x}+\mu A-(1+i \beta) A|A|^{2}+(\gamma+i \delta) A|B|^{2} \\
& B_{t}=(1+i \alpha) B_{x x}+\varepsilon-1 B_{x}+\mu B-(1+i \beta) B|B|^{2}+(\gamma+i \delta) B|A|^{2}
\end{aligned}
$$

$A(x+1, t)=A(x, t) \exp (-i a), \quad B(x+1, t)=B(x, t) \exp (i a)$ if $x \in \mathbb{R}, t>0$

$$
A(x, 0)=A_{0}(x), \quad B(x, 0)=B_{0}(x) \quad \text { if } x \in \mathbb{R} .
$$

Here $A$ and $B$ are appropriately smooth complex-valued functions of the spatial variable $x$ and the time $t ; i$ is the imaginary unit; the real parameters $\alpha, \beta, \gamma, \delta, \mu$, and $a$ are such that

$$
\mu>0, \quad \gamma<1, \text { and } 0 \leq a<2 \pi
$$

and the real parameter $\varepsilon>0$ is assumed to be small. The functions $A_{0}$ and $B_{0}$ appearing in the initial conditions (1.4) are appropriately smooth. Then, as we shall see in $§ 3$, the problem (1.1)-(1.4) is well posed and its solution is defined in $0<t<\infty$. In addition, we shall consider the problem (1.1)-(1.4) under one of the following additional conditions:

$$
\begin{aligned}
& A(x, t)=-B(-x, t) \quad \text { if } x \in \mathbb{R}, t \geq 0 \\
& A(x, t)=B(-x, t) \quad \text { if } x \in \mathbb{R}, t \geq 0
\end{aligned}
$$

*Received by the editors March 4, 1991; accepted for publication (in revised form) September 2, 1992. This research was partially supported by Direccion General de Investigación Cientifica y Técnica grant PB-90-0271.

tEscuela Técnica Superior de Ingenieros Aeronáuticos, Universidad Politécnica de Madrid, 28040 Madrid, Spain. 
As we shall see in $\$ 3$ each of these conditions is compatible with (1.1)-(1.4) in the following sense: if a solution of (1.1)-(1.4) satisfies that condition for $t=0$, then the condition holds for all $t>0$.

Equations (1.1), (1.2) appear as a normal form in a weakly nonlinear analysis of nonequilibrium systems at the onset of the so-called oscillatory instability; see [1]-[4]. In particular, they have been used in the analysis of wave dynamics in doubly diffusive and binary fluid convection (see [1], [5], [6]), and some of their solutions have been seen to describe qualitatively the results of some experiments (see [7]-[11]).

Let us now explain briefly how these equations are obtained and where conditions (1.3), (1.6), and (1.7) come from. We consider a system of PDEs of the form

$$
\frac{\partial u}{\partial T}=G\left(\frac{\partial^{2}}{\partial X^{2}}, u ; R\right) \text { in }-\infty<X<\infty, T>0,
$$

where $u=\left(u_{1}, \ldots, u_{N}\right)$ is a function of the space variable $X$, and the time variable $T$, $R$ is a control parameter, and $G$ is a nonlinear differential operator (invariant under space translations, $X \rightarrow X+c$, and reflection, $X \rightarrow-X)$ such that $G\left(\partial^{2} / \partial X^{2}, 0 ; R\right) \equiv$ 0 (the uniform state $u \equiv 0$ is a stationary solution of (1.8) for all $R$ ).

Let $\varphi\left(\omega, k^{2}, R\right)=0$ ( $\omega=$ growth rate, $k=$ wave number) be the complex dispersion relation of the linearized problem about $u=0$. We assume that the growth rate of the most unstable modes are of the form

$$
\begin{aligned}
& \omega= \pm i \Omega+c_{1 \pm} R \mp c_{2} k_{0}\left(k-k_{0}\right)-c_{3 \pm}\left(k-k_{0}\right)^{2}+o(|R|)+o\left(\left|k-k_{0}\right|^{2}\right), \\
& \omega= \pm i \Omega+c_{1 \pm} R \pm c_{2} k_{0}\left(k+k_{0}\right)-c_{3 \pm}\left(k+k_{0}\right)^{2}+o(|R|)+o\left(\left|k+k_{0}\right|^{2}\right),
\end{aligned}
$$

as $R \rightarrow 0$ and $k \rightarrow \pm k_{0}$, while $\operatorname{Re} \omega<0$ otherwise. Here the coefficients $\Omega$ and $k_{0}$ are real, while $c_{1 \pm}, c_{2}$ and $c_{3 \pm}$ are complex, and such that $c_{1-}=\bar{c}_{1+}, c_{3-}=\bar{c}_{3+}$ (overbars stand for the complex conjugate) and

$$
\Omega>0, \quad k_{0}>0, \quad \operatorname{Re} c_{1+}>0, \quad \operatorname{Re} c_{2}=0, \quad \operatorname{Rec} 3+>0 .
$$

Notice that the corresponding neutrally stable modes at $R=0$ are of the form

$$
u=U_{0}\left[A \exp \left(i \Omega T+i k_{0} X\right)+B \exp \left(i \Omega T-i k_{0} X\right)\right]+\text { c.c. }
$$

for a certain eigenvector $U_{0} \in \mathbb{C}^{N}$, where the complex amplitudes $A$ and $B$ are arbitrary and c.c. stands for the complex conjugate. That mode is the superposition of two wavetrains that are travelling in opposite directions. The weakly nonlinear stabil-" ity of those pairs of waves, as $R \rightarrow 0$, is analyzed by appropriately scaling the complex * amplitudes and allowing them to depend on the slow time and space variables $t=\varepsilon^{2} T$ and $x=\varepsilon X$, where the small parameter $\varepsilon>0$ is defined by $\varepsilon^{2}=R$. If the ansatz

$$
u=\varepsilon U_{0}\left[A(x, t) \exp \left(i \Omega T+i k_{0} X\right)+B(x, t) \exp \left(i \Omega T-i k_{0} X\right)\right]+\text { c.c. }+O\left(\varepsilon^{2}\right)
$$

is inserted into (1.8) and the appropriate solvability conditions are applied at the orders $O\left(\varepsilon^{2}\right)$ and $O\left(\varepsilon^{3}\right)$, then the following evolution equations are obtained (at the order $\left.O\left(\varepsilon^{3}\right)\right)$ :

$$
A_{t}=c_{3+} A_{x x}-\left(\frac{c_{2} k_{0}}{i \varepsilon}\right) A_{x}+c_{1+} A+c_{4} A|A|^{2}+c_{5} A|B|^{2}
$$




$$
B_{t}=c_{3+} B_{x x}+\left(\frac{c_{2} k_{0}}{i \varepsilon}\right) B_{x}+c_{1+} B+c_{4} B|B|^{2}+c_{5} B|A|^{2},
$$

where the coefficients $c_{1+}, c_{2}$, and $c_{3+}$ are as defined above, and $c_{4}$ and $c_{5}$ depend on the leading nonlinear terms of (1.8). Equations (1.11), (1.12) may be obtained quite directly by symmetry considerations (see [1]), but the actual values of the coefficients $c_{4}$ and $c_{5}$ must be obtained by the process described above (which usually leads to quite tedious calculations, even with the help of symbolic algebra). Notice that the coefficient $\left(c_{2} k_{0} / i \varepsilon\right)$ is real and large as $\varepsilon \rightarrow 0$ if $c_{2} \neq 0$ as we are assuming (the assumption $c_{2}=0$ would restrict the analysis to a codimension-two point of the parameter space of (1.8)). In addition to (1.9), the following supercriticality assumption will be made:

$$
\operatorname{Re} c_{4}<0, \quad \operatorname{Re}\left(c_{4}+c_{5}\right)<0 .
$$

In fact, if (1.13) does not hold, then (1.11), (1.12) possess solutions that either are unbounded as $t \rightarrow \infty$ or blow up in a finite time, as is readily seen by considering spatially uniform solutions.

Now, let us impose the following spatial periodicity condition on the solutions of (1.8):

$$
u(X+L, T) \equiv u(X, T),
$$

where the period $L$ depends on $\varepsilon$ in such a way that $\varepsilon L \rightarrow l>0$ and $2 \pi$ fract $\left(k_{0} L / 2 \pi\right) \rightarrow d$ as $\varepsilon \rightarrow 0$ (fract $(z)$ stands for the fractionary part of the real $z$ ). Then if condition (1.14) is imposed on (1.10), the following conditions are obtained (to the leading order):

$$
A(x+l, t) \equiv A(x, t) \exp (-i d), \quad B(x+l, t) \equiv B(x, t) \exp (i d) .
$$

Observe that the conditions (1.14) are appropriate to analyze the problem (1.8) in a finite interval, $0<X<L / 2$, if the boundary conditions at $X=0$ and $L / 2$ are either of the Dirichlet $(u=0)$ or Neumann $\left(u_{X}=0\right)$ type. This is readily seen when

* $u$ is extended to $-\infty<X<\infty$ by means of the appropriate reflexion principle:

$$
\begin{aligned}
& u(X+m L / 2, T) \equiv-u(-X+m L / 2, T) \quad \text { (Dirichlet), or } \\
& u(X+m L / 2, T) \equiv u(-X+m L / 2, T) \quad \text { (Neumann) }
\end{aligned}
$$

for each integer $m$. When (1.16) or (1.17) are imposed on (1.10), then (to leading order) we obtain (1.15) and

$$
\begin{gathered}
A(x, t) \equiv-B(-x, t) \text { (Dirichlet), or } \\
A(x, t) \equiv B(-x, t) \text { (Neumann). }
\end{gathered}
$$

Now, by applying in (1.11), (1.12), (1.15) an appropriate transformation of the type $A(x, t) \rightarrow A(x, t) \exp ($ iet $), B(x, t) \rightarrow B(x, t) \exp ($ iet $)$ (to make real the coefficients of $A$ and $B$ in (1.11) and (1.12), respectively), and rescaling the variables $A, B, x$, and $t$, and the small parameter $\varepsilon$, we obtain (1.1)-(1.3); conditions (1.5) readily follow from 
(1.13). By the same process, the additional condition (1.18) (respectively, (1.19)) leads to (1.6) (respectively, (1.7))

Therefore, the problem (1.1)-(1.4) seems to be appropriate to analyze spatially periodic patterns of (1.8) near the instability limit. Pattern formation in finite intervals, under homogeneous Dirichlet or Neumann boundary conditions, is analyzed by imposing (1.6) or (1.7).

The main object of this paper is to obtain rigorously the following approximate model (of (1.1)-(1.4)) in the limit $\varepsilon \rightarrow 0: A(x, t) \simeq V(x-t / \varepsilon, t), B(x, t) \simeq$ $W(x+t / \varepsilon, t)$, where the functions $(y, t) \rightarrow V(y, t)$ and $(z, t) \rightarrow W(z, t)$ satisfy, in first approximation,

$$
\begin{gathered}
V_{t}=(1+i \alpha) V_{y y}+\mu V-(1+i \beta) V|V|^{2}+(\gamma+i \delta) V\left\langle|W|^{2}\right\rangle \\
W_{t}=(1+i \alpha) W_{z z}+\mu W-(1+i \beta) W|W|^{2}+(\gamma+i \delta) W\left\langle|V|^{2}\right\rangle, \\
V(y+1, t)=V(y, t) \exp (-i a), \quad W(z+1, t)=W(z, t) \exp (i a), \\
V(y, 0)=A_{0}(y), \quad W(z, 0)=B_{0}(z),
\end{gathered}
$$

if $y, z \in \mathbf{R}, t>0$. Here, the spatial average $\langle\cdot\rangle$ is defined by

$$
\langle f\rangle=\int_{0}^{1} f(\xi, t) d \xi
$$

for each function $f \in C(\mathbf{R} \times[0, \infty[)$ that is periodic, of period 1 , in its first argument (as $|V|^{2}$ and $|W|^{2}$ are; see (1.22)). In addition, we shall see that if $A$ and $B$ satisfy one of the additional conditions, (1.6) or (1.7), then $\left\langle|W|^{2}\right\rangle=\left\langle|V|^{2}\right\rangle$ for all $t \geq 0$, and the model (1.20)-(1.23) may be further simplified to

$$
\begin{aligned}
& V_{t}=(1+i \alpha) V_{y y}+\mu V-(1+i \beta) V|V|^{2}+(\gamma+i \delta) V\left(|V|^{2}\right\rangle \\
& V(y+1, t)=V(y, t) \exp (-i a), V(y, 0)=A_{0}(y) \text { if } y \in \mathbf{R}, t>0 .
\end{aligned}
$$

These two models were first obtained, independently, by Knobloch and De Luca [12] and by Alvarez-Pereira and Vega [13]. In both cases a formal derivation was made, by means of perturbation techniques. In fact, in [13] the problem under consideration was not of the type (1.8), but (1.20)-(1.23) and (1.25)-(1.26) appeared in a weakly nonlinear stability analysis, in two space dimensions, of travelling plane wave-fronts in a reaction-diffusion system arising in combustion theory; that problem is essentially more involved than (1.8), and the models $(1.20)-(1.23)$ and $(1.25)-(1.26)$ were obtained in a particular limit.

The paper is organized as follows. For convenience, a formal derivation (by means of a two-time scales method) of (1.20)-(1.23) is given in $\S 2$. Section 3 includes some preliminaries concerning the model (1.1)-(1.4), a rigorous derivation of the approximate models and some basic properties of these models. Finally, in $\$ 4$ we describe further properties of the approximate models, and make some conjectures. 
2. A formal derivation of the model (1.20)-(1.23). Here we give a formal derivation of model (1.20)-(1.23) by means of perturbation techniques (the second asymptotic model is easily obtained from the first one, as it will be seen in §3). That derivation will give the key idea for the rigorous analysis of next section. In addition, it will provide some insight into the nature of the limit $\varepsilon \rightarrow 0$, by explaining why it is natural to expect the appearance of the spatial averaged terms in the approximate models.

As $\varepsilon \rightarrow 0$, there are two obvious time scales in (1.1), (1.2):

$$
t \sim 1 \text { and } \tau \equiv t / \varepsilon \sim 1 .
$$

The latter comes when balancing $A_{t}$ with $-\varepsilon^{-1} A_{x}$ in (1.1) (or $B_{\imath}$ with $\varepsilon^{-1} B_{x}$ in (1.2)). Then we shall seek the expansions

$$
A(x, t)=\tilde{A}_{0}(x, \tau, t)+\varepsilon \tilde{A}_{1}(x, \tau, t)+\cdots, \quad B(x, t)=\tilde{B}_{0}(x, \tau, t)+\varepsilon \tilde{B}_{1}(x, \tau, t)+\cdots .
$$

When these expansions are inserted into (1.1)-(1.3), and the coefficients of $\varepsilon^{0}$ and $\varepsilon^{1}$ are set to zero, the following problems are obtained:

$$
\tilde{A}_{0 \tau}+\tilde{A}_{0 x}=0, \quad \tilde{B}_{0 \tau}-\tilde{B}_{0 x}=0,
$$

$$
\tilde{A}_{1 \tau}+\tilde{A}_{1 x}=-\tilde{A}_{0 t}+(1+i \alpha) \tilde{A}_{0 x x}+\tilde{A}_{0}\left[\mu-(1+i \beta)\left|\tilde{A}_{0}\right|^{2}+(\gamma+i \delta)\left|\tilde{B}_{0}\right|^{2}\right]
$$

$$
\tilde{B}_{1 \tau}-\tilde{B}_{1 x}=-\tilde{B}_{0 t}+(1+i \alpha) \tilde{B}_{0 x x}+\tilde{B}_{0}\left[\mu-(1+i \beta)\left|\tilde{B}_{0}\right|^{2}+(\gamma+i \delta)\left|\tilde{A}_{0}\right|^{2}\right]
$$

$$
\tilde{A}_{j}(x+1, \tau, t) \equiv \tilde{A}_{j}(x, \tau, t) \exp (-i a), \quad \tilde{B}_{j}(x+1, \tau, t) \equiv B_{j}(x, \tau, t) \exp (i a)
$$

$$
\tilde{A}_{0}(x, 0,0) \equiv A_{0}(x), \quad \tilde{B}_{0}(x, 0,0) \equiv B_{0}(x), \quad \tilde{A}_{1}(x, 0,0) \equiv \tilde{B}_{1}(x) \equiv 0
$$

for $j=0$ and 1 . The wave equations (2.2) readily yield $\tilde{A}_{0}(x, \tau, t) \equiv V_{0}(x-\tau, t)$, $\vec{B}_{0}(x, \tau, t) \equiv W_{0}(x+\tau, t)$, for some functions $(y, t) \rightarrow V_{0}(y, t)$ and $(z, t) \rightarrow W_{0}(z, t)$ that satisfy (1.22), (1.23) (see (2.5), (2.6)) and are otherwise arbitrary (at the moment). Then the solution of (2.3), (2.4) may be found in close-form

$$
\begin{gathered}
\tilde{A}_{1}(x, \tau, t)=V_{1}(x-\tau, t)+\tau\left[-V_{0 t}+(1+i \alpha) V_{0 y y}+V_{0}\left(\mu-(1+i \beta)\left|V_{0}\right|^{2}\right)\right] \\
+\left(\frac{\gamma+i \delta}{2}\right) V_{0} \int_{0}^{\tau+x}\left|W_{0}(z, t)\right|^{2} d z
\end{gathered}
$$

$$
\begin{aligned}
& \tilde{B}_{1}(x, \tau, t)=W_{1}(x+\tau, t)+\tau\left[-W_{0 t}+(1+i \alpha) W_{0 z z}+W_{0}\left(\mu-(1+i \beta)\left|W_{0}\right|^{2}\right)\right] \\
& \quad+\left(\frac{\gamma+i \delta}{2}\right) W_{0} \int_{0}^{\tau-x}\left|V_{0}(y, t)\right|^{2} d y
\end{aligned}
$$

for some functions $V_{1}$ and $W_{1}$ (to be determined at later stages). 
Now, by eliminating secular terms in the fast time scale, i.e., by requiring the right-hand side of (2.7) to be bounded as $\tau \rightarrow \infty(t=$ constant in this time scale) we readily obtain

$$
\begin{aligned}
-V_{0 t}+(1+i \alpha) V_{0 y y} & +V_{0}\left[\mu-(1+i \beta)\left|V_{0}\right|^{2}\right] \\
& +(\gamma+i \delta) V_{0} \lim _{\tau \rightarrow \infty}(2 \tau)^{-1} \int_{0}^{\tau+x}\left|W_{0}(z, t)\right|^{2} d z=0 .
\end{aligned}
$$

Notice that for each fixed value of $y=x-\tau$, the first three terms in the lefthand side of this equation are constant (recall that $t=$ constant at this time stage), while the last one is of the form $(\gamma+i \delta) V_{0} \lim _{\tau \rightarrow \infty}(2 \tau)^{-1} \int_{0}^{2 \tau+y}\left|W_{0}(z, t)\right|^{2} d z$, and since $\left|W_{0}(z+1, t)\right|^{2} \equiv\left|W_{0}(z, t)\right|^{2}$ (see $(1.22)$ ), we have $(2 \tau)^{-1} \int_{0}^{2 \tau+y}\left|W_{0}(z, t)\right|^{2} d z=$ $\left\langle\left|W_{0}\right|^{2}\right\rangle+O\left(\tau^{-1}\right)$ as $\tau \rightarrow \infty$, where the spatial average $\langle\cdot\rangle$ was defined in (1.24). Then $V_{0}$ and $W_{0}$ satisfy (1.20). Equation (1.21) is obtained in a completely similar way, by eliminating secular terms in (2.8), and the derivation of $(1.20)-(1.23)$ is complete.

Observe that, to the leading order, in the fast time scale $(\tau \sim 1)$, the amplitudes $A$ and $B$ satisfy the wave equations (2.2), whose solution represents two waves that are travelling in opposite directions. If one moves in a reference frame attached to the wave associated with $A$ (i.e., if $x-\tau=$ constant), then the wave associated with $B$ is seen to travel at a speed 2 in the fast time scale, or at a sped $2 / \varepsilon(\rightarrow \infty$ as $\varepsilon \rightarrow 0$ ) in the slow time scale. Therefore, if $t \sim 1$, then the spatial structure of $B$ is not appreciated from the reference frame moving with $A$; only the spatial mean value of $|B|^{2}$ over a period is seen. This explains the appearance of the spatial averages in the asymptotic equations, and suggests the main argument in the proof of Theorem 3.4 below.

3. Main results concerning the models (1.1)-(1.4), (1.20)-(1.23), and (1.25)-(1.26). Here we first consider sorne preliminary properties of the model (1.1)(1.4). Then, the approximate models $(1.20)-(1.23)$ and $(1.25)-(1.27)$ are rigorously derived. Finally, some basic properties of the approximate models are given.

3.1. The model (1.1)-(1.4). In order to prove that the model (1.1) $-(1.4)$ is well posed we could modify appropriately an abstract result by Ghidaglia [14] that was used be Temam [15] to prove the well-posedness of the standard complex cubic Ginzburg-Landau equation in finite domains with standard boundary conditions. Nevertheless, for the sake of brevity, we shall follow a more direct approach, based on a classical result by Henry [16].

THEOREM 3.1. If $\gamma<1$ and if the functions $A_{0}$ and $B_{0}$ belong to (the complexified space of $C^{2+\alpha}([0,1])$, for some $\left.\alpha \in\right] 0,1[$, and satisfy (1.3), then (1.1)-(1.4) possess a unique classical solution, $(x, t) \rightarrow(A(x, t), B(x, t))$, such that $A, B \in C^{2+\alpha, 1+\alpha / 2}([0,1]$ $\times[0, T])$ for all $T>0$. In addition, $A, B \in C^{2 k, k}\left([0,1] \times\left[T_{1}, T_{2}\right]\right)$ whenever $0<T_{1}<T_{2}$ and $k \geq 0$.

Proof. We first state (1.1)-(1.4) in an appropriate abstract setting (after decomplexification and restriction to the bounded interval $0 \leq x \leq 1$, with the appropriate boundary conditions at $x=0,1$, obtained from (1.3)) as

$$
\frac{d u}{d t}+L u=f(u) \text { if } t>0, \quad u(0)=u_{0} \in D(L),
$$

where the linear operator $L: D(L) \rightarrow X \equiv L_{2}(10,10)^{4}$ is defined in such a way that $f: X \rightarrow X$ is a substitution perator, and

$$
D(L)=\left\{u \in H_{2}(] 0,1[)^{4}: u \text { satisfies the boundary conditions at } x=0,1\right\}
$$


Then the operator $L$ is sectorial in $X$, and $f$ is locally Lipschitzian and maps bounded sets into bounded sets. Then, by applying $[17, \mathrm{p}$. 55, Thm. 3.3.4], it follows that (3.1) possesses a unique mild solution, $u \in C([0, T[, X)$, in a maximal interval of existence, $0 \leq t<T$, and one of the following alternatives holds: either (i) $T=\infty$, or (ii) $T<\infty$ and there is a sequence, $\left\{t_{m}\right\} \subset \mathbb{R}$, such that $t_{m} \rightarrow T^{-}$and $\left\|u\left(t_{m}\right)\right\|_{X} \rightarrow \infty$. But since $\gamma<1$, according to the result in Lemma 3.3 below (that may be seen as an a priori estimate on (3.1)) the alternative (ii) cannot hold. Then $T=\infty$, and we obtain global existence and uniqueness of mild solutions. Further regularity of $u$ is obtained as usual, by means of parabolic estimates [17, VII-10] (those estimates are readily extended to apply for boundary conditions such as (1.3)) and imbedding theorems $[17$, II-3].

Now we prove that the additional conditions (1.6) and (1.7) are compatible with the model (1.1)-(1.4).

LEMMA 3.2. If a classical solution of (1.1)-(1.4) satisfies (1.6) (respectively, (1.7)) at $t=0$, then (1.6) (respectively, (1.7)) holds for all $t>0$.

Proof. Let $(A, B)$ be a classical solution of $(1.1)-(1.4)$ satisfying (1.6) (if it satisfies (1.7) the argument is similar) at $t=0$, and let the pair of functions $A_{1}$ and $B_{1}$ be defined by $A_{1}(x, t) \equiv-B(-x, t)$ and $B_{1}(x, t) \equiv-A(-x, t)$. The pair $\left(A_{1}, B_{1}\right)$ satisfies (1.1)-(1.3), and since (1.6) holds at $t=0, A_{1}(x, 0)=A(x, 0)$ and $B_{1}(x, 0)=B(x, 0)$ for all $x \in \mathbb{R}$. Then, by uniqueness of (1.1)-(1.4), $A_{1}(x, t)=A(x, t)$ and $B_{1}(x, t)=B(x, t)$ for all $x \in \mathbf{R}$ and all $t>0$, i.e., condition (1.6) holds for all $t>0$, as stated.

3.2. A rigorous derivation of the approximate models. As suggested by the formal derivation in $\$ 2$, the approximate models will be obtained, in Theorem 3.4, by means of an averaging method. To apply that method we need some estimates on (1.1)-(1.4) that hold uniformly as $\varepsilon \rightarrow 0$ (see Lemma 3.5 below). Two basic inequalities that will be used systematically in the proof of Lemma 3.3 are first given.

If $u \in C^{1}([0,1] \times \mathbb{R})$ is such that $\int_{0}^{1} u(x) d x=0$, then

$$
\|u\|_{L_{q}(0,10)} \leq k_{q r}\left\|u_{x}\right\|_{L_{r}(00,10)}^{\alpha} \cdot\|u\|_{\left.L_{r}(00,1]\right)}^{1-\alpha},
$$

whenever $g>r \geq 1$, where $\alpha=1 / r-1 / q$, and the constant $k_{q r}$ depends only on $q$ and $r$ (it is independent of $u)$. If $v \in C^{\mathbf{1}}([0,1], \mathbb{C})$ and $q>1$, then

$$
\|v\|_{L_{2 q}(0,1)}^{2} \leq\|v\|_{L_{2}(0,1)}^{2}+2 k_{q 1}\|v\|_{L_{2}(0,10)}^{1+1 / q} \cdot\left\|v_{x}\right\|_{\left.L_{2}(00,1]\right)}^{1-1 / q},
$$

where the constant $k_{q 1}$ is defined as in (3.2).

The inequality (3.2) is given in, e.g., [17, pp. 62-63], while (3.3) is obtained by applying (3.2) (and Hölder's inequality) to the function $x \rightarrow u(x) \equiv|v(x)|^{2}-$ $\|v\|_{L_{2}(0,1)}^{2}$.

We now give some uniform estimates on the solutions of (1.1)-(1.4). For the sake of brevity, we shall not try to obtain the best possible values of the bounding constants $K_{0}, T$, and $C_{0}$. Notice that these constants are independent of $\varepsilon$, that $C_{0}$ is also independent of the particular solution of (1.1)-(1.4), and that $K_{0}$ and $T$ depend on it only through $\phi_{j}(0)$.

LEMMA 3.3. If $\gamma<1$, for each classical solution of (1.1)-(1.4) and for $j=$ 0,1 , and 2 , let the functions $t \rightarrow \phi_{j}(t)$ be defined by

$$
\phi_{j}(t) \equiv \int_{0}^{1}\left[\left|\frac{\partial^{j} A}{\partial x^{j}}\right|^{2}+\left|\frac{\partial^{j} B}{\partial x^{j}}\right|^{2}\right] d x .
$$


Then, for $j=0,1$, and $2, \phi_{j}$ satisfies

$$
\phi_{j}(t) \leq K_{0} \quad \text { for all } t \geq 0, \quad \text { and } \phi_{j}(t) \leq C_{0} \quad \text { for all } t \geq T \text {, }
$$

where the constants $K_{0}$ and $T$ (respectively, $C_{0}$ ) depend only on $\beta, \gamma, \delta, \mu, \phi_{1}(0)$, $\phi_{2}(0)$, and $\phi_{3}(0)$ (respectively, on $\beta, \gamma, \delta$, and $\mu$ ).

Proof. The estimates (3.5) will be obtained successively for $\phi_{0}, \phi_{1}$, and $\phi_{2}$. For the sake of clarity we shall simplify the notation as follows. Every constant appearing below that depends only on $\beta, \gamma, \delta, \mu, \phi_{1}(0), \phi_{2}(0)$, and $\phi_{3}(0)$ (respectively, on $\beta, \gamma$, $\delta$, and $\mu$ ) will be denoted always as $K$ (respectively, as $C$ ).

Step 1. The estimates (3.5) for $\phi_{0}$. Let us multiply (1.1) by $\bar{A},(1.2)$ by $\bar{B}$, add the resulting equations, take the real part, integrate on $0<x<1$, integrate by parts, use (1.3), and apply Hölder's inequality twice to obtain

$$
\frac{d \phi_{0}}{d t}=-2 \phi_{1}+2 \mu \phi_{0}-2 \int_{0}^{1}\left[|A|^{4}+|B|^{4}-2 \gamma|A|^{2}|B|^{2}\right] d x
$$

$$
\leq-2 \phi_{1}+2 \mu \phi_{0}-2 C \int_{0}^{1}\left[|A|^{4}+|B|^{4}\right] d x \leq-2 \phi_{1}+2 \mu \phi_{0}-C \phi_{0}^{2} \quad \text { if } t>0,
$$

where $C=\min \{1,1-\gamma\}>0$. Since $\phi_{1}(t) \geq 0$ for all $t>0$, the estimates (3.5) readily follow (with, e.g., $K_{0}=\max \left\{\phi_{0}(0), 2 \mu / C\right\}, C_{0}=4 \mu / C$ and $T=(2 \mu)^{-1} \log [\max \{1,2-$ $\left.\left.4 \mu / C \phi_{0}(0)\right\}\right]$ if $\mu>0$, while $K_{0}=\phi_{0}(0), C_{0}=1$, and $T=\max \left\{0,\left(\phi_{0}(0)-1\right) / C \phi_{0}(0)\right\}$ if $\mu \leq 0$ ).

Step 2. The estimates (3.5) for $\phi_{1}$. Now, we multiply (1.1) by $-\bar{A}_{x x},(1.2)$ by $-\bar{B}_{x x}$, add the resulting equations, take the real part, integrate on $0<x<1$, integrate by parts and use (1.3). Then, the following equation follows:

$$
\begin{aligned}
\frac{d \phi_{1}}{d t}= & -2 \phi_{2}+2 \mu \phi_{1} \\
& -\int_{0}^{1}\left\{2|A|^{2}\left|A_{x}\right|^{2}+2|B|^{2}\left|B_{x}\right|^{2}+\left[\left(|A|^{2}\right)_{x}\right]^{2}+\left[\left(|B|^{2}\right)_{x}\right]^{2}\right\} d x+F_{1}+F_{2}
\end{aligned}
$$

for all $t>0$, where

$$
\begin{aligned}
F_{1} & =-\gamma \int_{0}^{1}\left[\left(A \bar{A}_{x x}+\bar{A} A_{x x}\right)|B|^{2}+\left(B \bar{B}_{x x}+\bar{B} B_{x x}\right)|A|^{2}\right] d x \\
& \leq 4|\gamma|\left[\int_{0}^{1}\left(|A|^{6}+|B|^{6}\right) d x \int_{0}^{1}\left(\left|A_{x x}\right|^{2}+\left|B_{x x}\right|^{2}\right) d x\right]^{1 / 2}, \\
F_{2} & =i \int_{0}^{1}\left[\left(A \bar{A}_{x x}-\bar{A} A_{x x}\right)\left(\beta|A|^{2}-\delta|B|^{2}\right)+\left(B \bar{B}_{x x}-\bar{B} B_{x x}\right)\left(\beta|B|^{2}-\delta|A|^{2}\right] d x\right. \\
& \leq 4(|\beta|+|\delta|)\left[\int_{0}^{1}\left(|A|^{6}+|B|^{6}\right) d x \int_{0}^{1}\left(\left|A_{x x}\right|^{2}+\left|B_{x x}\right|^{2}\right) d x\right]^{1 / 2},
\end{aligned}
$$

or, by using (3.3) (with $q=3$ ), $F_{1}+F_{2} \leq C \phi_{0}(t)\left[\phi_{0}(t)+\phi_{1}(t)\right]^{1 / 2} \phi_{2}(t)^{1 / 2}$ if $t>0$, where the constant $C$ is as defined above (in fact, $C$ depends only on $\beta, \gamma$, and $\delta$ ). Then, (3.7) yields $d \phi_{1} / d t \leq-2 \phi_{2}+2 \mu \phi_{1}+C \phi_{0}\left(\phi_{0}+\phi_{1}\right)^{1 / 2} \phi_{2}^{1 / 2}$ if $t>0$, and since 
the function $z \rightarrow f(z) \equiv-z+c z^{1 / 2}$ ( $c \geq 0$ being a constant) satisfies $f(z) \leq c^{2} / 4$ for all $z \geq 0$, we have

$$
\frac{d \phi_{1}}{d t} \leq-\phi_{2}+2 \mu \phi_{1}+\frac{C^{2} \phi_{0}^{2}\left(\phi_{0}+\phi_{1}\right)}{4} \quad \text { if } t>0
$$

Then, if the constant $K>0$ is such that $2 \mu+C^{2} \phi_{0}(t)^{2} / 4 \leq K$ for all $t>0$, we multiply (3.6) by $K$ and add the resulting equation to (3.8) to obtain $d\left(\phi_{1}+K \phi_{0}\right) / d t \leq$ $-K\left(\phi_{1}+K \phi_{0}\right)+K(K+2 \mu) \phi_{0}+C \phi_{0}^{3} / 4$ if $t>0$, and the first estimate (3.5) readily follows for $\phi_{1}$. The second estimate (3.5) is obtained in a similar way, when taking into account that it holds for $\phi_{0}$, and using an appropriate linear combination of the inequalities (3.6) and (3.8).

Step 3. The estimates (3.5) for $\phi_{2}$. Again, we shall use the following equation, which is obtained by differentiating (1.1) and (1.2) with respect to $x$, multiplying the resulting equations by $-\bar{A}_{x x x}$ and $-\bar{B}_{x x x}$, respectively, adding, taking the real part, integrating on $0<x<1$, integrating by parts, and using (1.3):

$$
\frac{d \phi_{2}}{d t}=-2 \phi_{3}+2 \mu \phi_{2}+F_{3}+F_{4} \text { if } t>0
$$

where $\phi_{3}$ is defined as in (3.4) and (recall that c.c. stands for the complex conjugate)

$$
\begin{gathered}
F_{3}=(1+i \beta) \int_{0}^{1}\left[\bar{A}_{x x x}\left(A|A|^{2}\right)_{x}+\bar{B}_{x x x}\left(B|B|^{2}\right)_{x}\right] d x+\text { c.c. } \\
\leq 6|1+\beta| \int_{0}^{1}\left[|A|^{2}\left|A_{x}\right|\left|A_{x x x}\right|+|B|^{2}\left|B_{x}\right|\left|B_{x x x}\right|\right] d x \\
F_{4}=-(\gamma+i \delta) \int_{0}^{1}\left[\bar{A}_{x x x}\left(A|B|^{2}\right)_{x}+\bar{B}_{x x x}\left(B|A|^{2}\right)_{x}\right] d x+c . c . \\
\leq 2|\gamma+i \delta| \int_{0}^{1}\left[\left(2|A|\left|B_{x}\right|+|B|\left|A_{x}\right|\right)|B|\left|A_{x x x}\right|\right. \\
\left.+\left(2|B|\left|A_{x}\right|+|A|\left|B_{x}\right|\right)|A|\left|B_{x x x}\right|\right] d x .
\end{gathered}
$$

But, according to the mean value theorem, if $t>0$, then $|A(x, t)|^{2} \leq 2 \phi_{0}(t)+\phi_{1}(t)$, and $|B(x, t)|^{2} \leq 2 \phi_{0}(t)+\phi_{1}(t)$. Then, by applying Hölder's inequality we readily obtain $F_{3}+F_{4} \leq C\left[\phi_{0}(t)+\phi_{1}(t)\right] \phi_{1}(t)^{1 / 2} \phi_{3}(t)^{1 / 2}$ for all $t>0$, where the constant $C$ is as defined at the beginning of the proof (in fact, it depends only on $\beta, \gamma$, and $\delta$ ). Then, (3.9) yields $d \phi_{2} / d t=-2 \phi_{3}+2 \mu \phi_{2}+C\left(\phi_{0}+\phi_{1}\right) \phi_{1}^{1 / 2} \phi_{3}^{1 / 2}$ if $t>0$, and by the argument leading to (3.8) above,

$$
\frac{d \phi_{2}}{d t} \leq 2 \mu \phi_{2}+\frac{C^{2}\left(\phi_{0}+\phi_{1}\right)^{2} \phi_{1}}{4} \text { if } t>0
$$

Now, if (3.8) is multiplied by $\lambda=1+2|\mu|>0$, the resulting equation is added to (3.10) and the first estimate (3.5) for $\phi_{0}$ and $\phi_{1}$ is used, then the following inequality is obtained: $d\left(\phi_{2}+\lambda \phi_{1}\right) / d t \leq-\left(\phi_{2}+\lambda \phi_{1}\right)+K$ if $t>0$, where the constant $K$ is as defined at the beginning of the proof. By using this inequality, the first estimate (3.5) readily follows for $\phi_{2}$. Again, the second estimate (3.5) is obtained similarly, when taking into account that it holds for $\phi_{0}$ and $\phi_{1}$, and using the appropriate linear combination of (3.8) and (3.10). Thus, the proof is complete.

Now, we show that if $\varepsilon$ is sufficiently small, then the solutions of (1.1)-(1.4) satisfy approximately (1.20)-(1.23), in an appropriate uniform sense. 
THEOREM 3.4. If $\gamma<1$ and $0<\varepsilon<1$, let $(A, B)$ be a classical solution of (1.1)-(1.3) in $-\infty<x<\infty, t \geq 0$, and let the functions $V$ and $W$ be defined by $V(y, t) \equiv \varepsilon^{-4 / 5} \int_{t}^{t+\varepsilon^{4 / 5}} A(y+\tau / \varepsilon, \tau) d \tau, W(z, t) \equiv \varepsilon^{-4 / 5} \int_{t}^{t+\varepsilon^{4 / 5}} B(z-\tau / \varepsilon, \tau) d \tau$.

Then, for all $y, z \in \mathbf{R}$, and $t \geq 0, V$ and $W$ satisfy

$$
\begin{gathered}
V_{t}=(1+i \alpha) V_{y y}+\mu V-(1+i \beta) V|V|^{2}+(\gamma+i \delta) V\left\langle|W|^{2}\right\rangle+\varphi_{1}(y, t), \\
W_{t}=(1+i \alpha) W_{z z}+\mu W-(1+i \beta) W|W|^{2}+(\gamma+i \delta) W\left\langle|V|^{2}\right\rangle+\varphi_{2}(z, t), \\
V(y+1, t)=V(y, t) \exp (-i a), \quad W(z+1, t)=W(z, t) \exp (i a) \\
V(y, t)=A(y+t / \varepsilon, t)+\psi_{1}(y, t), \quad W(z, t)=B(z-t / \varepsilon, t)+\psi_{2}(z, t),
\end{gathered}
$$

where the spatial average $\langle\cdot\rangle$ is defined by (1.24) and the functions $\varphi_{j}$ and $\psi_{j}$ satisfy, for $j=1$ and 2 ,

$$
\begin{array}{rr}
\left\|\varphi_{j}(\cdot, t)\right\|_{\infty}, \quad\left\|\psi_{j}(\cdot, t)\right\|_{\infty} \leq K_{0} \varepsilon^{1 / 5}, & \text { if } \dot{t} \geq 0 \\
\left\|\varphi_{j}(\cdot, t)\right\|_{\infty}, \quad\left\|\psi_{j}(\cdot, t)\right\|_{\infty} \leq C_{0} \varepsilon^{1 / 5} & \text { if } t \geq T
\end{array}
$$

for some constants $K_{0}, T$, and $C_{0}$ such that $K_{0}$ and $T$ (respectively, $C_{0}$ ) depend only on $\|A(\cdot, 0)\|_{H^{2}(j 0,1]},\|B(\cdot, 0)\|_{H^{2}(\mid 0,1)}, \beta, \gamma, \delta$, and $\mu$ (respectively, on $\beta, \gamma, \delta$, and $\mu$ ). Here $H^{2}\left(j 0,1[)\right.$ is the usual (complexified) Sobolev space and $\|\cdot\|_{\infty}$ is the sup norm (if $g \in C^{2}(\mathbf{R})$, then $\left.\|f\|_{H^{2}(0,1)}=\mid \int_{0}^{1}\left(|f(\xi)|^{2}+\left|f^{\prime}(\xi)\right|^{2}+\left|f^{\prime \prime}(\xi)\right|^{2}\right) d \xi\right]^{1 / 2}$ and $\left.\|g\|_{\infty}=\sup \{|g(\xi)|: \xi \in \mathbf{R}\}\right)$.

Proof. We shall only obtain the estimate (3.15) (the proof below extends straightforwardly to obtain (3.16), when using the second estimate (3.5) of Lemma 3.3). As in the proof of Lemma 3.3 , every constant depending only on $\|A(\cdot, 0)\|_{H^{2}(0,1)}$, $\|B(\cdot, 0)\|_{\left.H^{2}(0,1]\right)}, \beta, \gamma, \delta$, and $\mu$ will be denoted always as $K$.

Let the functions $v$ and $w$ be defined by

$$
v(y, t) \equiv A(y+t / \varepsilon, t), \quad w(z, t) \equiv B(z-t / \varepsilon, t),
$$

which are readily seen to satisfy, for all $y, z \in \mathbb{R}$ and all $t \geq 0$,

$$
\begin{gathered}
v_{t}=(1+i \alpha) v_{y y}+\mu v-(1+i \beta) v|v|^{2}+(\gamma+i \delta) v|w(y-2 t / \varepsilon, t)|^{2} \\
w_{t}=(1+i \alpha) w_{z z}+\mu w-(1+i \beta) w|w|^{2}+(\gamma+i \delta) w|v(z+2 t / \varepsilon, t)|^{2} \\
v(y+1, t)=v(y, t) \exp (-i a), \quad w(z+1, t)=w(z, t) \exp (i a) .
\end{gathered}
$$

Also, according to Lemma 3.5 (see also (3.17) and (3.20)),

$$
\int_{0}^{1}\left(|v|^{2}+\left|v_{y}\right|^{2}+\left|v_{y y}\right|^{2}\right) d y+\int_{0}^{1}\left(|w|^{2}+\left|w_{z}\right|^{2}+\left|w_{z z}\right|^{2}\right) d z \leq K \quad \text { if } t \geq 0,
$$




$$
\|v(\cdot, t)\|_{\infty}+\|w(\cdot, t)\|_{\infty} \leq K \quad \text { if } t>0
$$

for some constant $K$, where the second inequality follows the first one. Then (3.18), (3.19) implies that $\int_{0}^{1}\left|v_{t}\right|^{2} d y+\int_{0}^{1}\left|w_{t}\right|^{2} d z \leq K$ for all $t>0$, for some constant $K$, and (see also (3.20)) for all $y_{0}, z_{0} \in \mathbf{R}$, and all $t_{0} \geq 0$,

$$
\begin{aligned}
& \int_{t_{0}}^{t_{0}+1}\left[\int_{y_{0}}^{y_{0}+1}\left(|v|^{2}+\left|v_{y}\right|^{2}+\left|v_{y y}\right|^{2}+\left|v_{t}\right|^{2}\right) d y\right] d t \leq K, \\
& \int_{t_{0}}^{t_{0}+1}\left[\int_{z_{0}}^{z_{0}+1}\left(|w|^{2}+\left|w_{z}\right|^{2}+\left|w_{z z}\right|^{2}+\left|w_{t}\right|^{2}\right) d z\right] d t \leq K
\end{aligned}
$$

for some constant $K$. Finally, by imbedding theorems (the Sobolev space $H^{2,1}\left(\mid a, b[\times] t_{0}, t_{0}+1[)\right.$ is continuously imbedded into the Hölder space $C^{1 / 2,1 / 4}([a, b]$ $\left.x\left[t_{0}, t_{0}+1\right]\right)$; see, e.g., $[11$, II-3]) we have

$$
\left|v\left(y, t_{2}\right)-v\left(y, t_{1}\right)\right|+\left|w\left(z, t_{2}\right)-w\left(z, t_{1}\right)\right| \leq K\left(t_{2}-t_{1}\right)^{1 / 4}
$$

if $y, z \in \mathbb{R}, 0 \leq t_{1} \leq t_{2} \leq t_{1}+1$, and this estimate and (3.21) are sufficient to obtain the required result.

To see that, first notice that since

$$
V(y, t)=\varepsilon^{-4 / 5} \int_{t}^{t+\varepsilon^{4 / 5}} v(y, \tau) d \tau, \quad W(z, t)=\varepsilon^{-4 / 5} \int_{t}^{t+e^{4 / 5}} w(z, \tau) d \tau,
$$

(3.20) readily implies that (3.13) holds. Also, if $0<\varepsilon<1$, then by using (3.22) we obtain, for all $y \in \mathbf{R}$ and all $t \geq \mathbf{0}$,

$$
\begin{aligned}
\left|\psi_{1}(y, t)\right| & =\varepsilon^{-4 / 5}\left|\int_{t}^{t+e^{4 / 5}}(v(y, \tau)-v(y, t)) d t\right| \\
& \leq K \varepsilon^{-4 / 5} \int_{t}^{t+\varepsilon^{4 / 5}}(t-\tau)^{1 / 4}=(4 K / 3) \varepsilon^{1 / 5},
\end{aligned}
$$

and the estimate (3.15) holds for $\psi_{1}$ (similarly it is seen that this estimate holds for $\psi_{2}$ ). Finally, to obtain the estimate (3.15) for $\varphi_{1}$ (it is obtained for $\varphi_{2}$ a similar way) observe that $\varphi_{1}$ is given by

$$
\varphi_{1}(y, t)=(1+i \beta) \varepsilon^{-4 / 5} F_{1}+(\gamma+i \delta) \varepsilon^{-4 / 5}\left(F_{2}+F_{3}+F_{4}\right),
$$

as obtained when (3.18) is integrated in $] t, t+\varepsilon^{4 / 5}[$, and the resulting equation is multiplied by $\varepsilon^{-4 / 5}$, where

$$
\begin{aligned}
& F_{1} \equiv \int_{t}^{t+\varepsilon^{4 / 5}} v(y, \tau)|v(y, \tau)|^{2} d \tau-\varepsilon^{4 / 5} V(y, t)|V(y, t)|^{2}, \\
& F_{2} \equiv \int_{t}^{t+\varepsilon^{4 / 5}}[V(y, t)-v(y, \tau)]|w(y-2 \tau / \varepsilon, \tau)|^{2} d \tau, \\
& F_{3} \equiv V(y, t) \int_{t}^{t+\varepsilon^{4 / 5}}\left\{|w(y-2 \tau / \varepsilon, \tau)|^{2}-|w(y-2 \tau / \varepsilon, t)|^{2}\right\} d \tau, \\
& F_{4} \equiv V(y, t)\left[\varepsilon^{4 / 5}\left\langle|W(\cdot, t)|^{2}\right\rangle-\int_{t}^{t+\varepsilon^{4 / 5}}|w(y-2 \tau / \varepsilon, t)|^{2} d \tau\right] .
\end{aligned}
$$


Then, to end up the proof, we only need to show that

$$
\left|F_{j}\right| \leq K \varepsilon \quad \text { if } t \geq 0 \text { for } j=1, \ldots, 4 \text {. }
$$

But the first three estimates (3.26) are readily obtained, by the argument leading to (3.24), when using (3.21) and (3.22), while the fourth one comes from the following expression, which is obtained by means of (3.20):

$$
\begin{aligned}
\int_{t}^{t+\varepsilon^{4 / s}} & |w(y-2 \tau / \varepsilon, t)|^{2} d \tau \\
& =(\varepsilon / 2) \int_{y-2 t / \varepsilon}^{y-2 t / \varepsilon-2 \varepsilon^{-1 / s}}|w(\xi, y)|^{2} d \xi \\
& =(\varepsilon / 2) \operatorname{Int}\left(2 \varepsilon^{-1 / 5}\right)\left\langle|W(\cdot, t)|^{2}\right\rangle-(\varepsilon / 2) \int_{0}^{\text {Fract }\left(2 \varepsilon^{-1 / 5}\right)}|w(\xi, t)|^{2} d \xi
\end{aligned}
$$

where Int and Fract stand for the integral and fractionary parts, respectively. When (3.26) is substituted into (3.25), the estimate (3.15) is obained for $\varphi_{1}$. Thus, the proof is complete.

Finally, if $\varepsilon$ is sufficiently small, then the solutions of (1.1)-(1.4) that satisfy one of the additional conditions, (1.6) or (1.7), are approximate solutions of (1.25) -(1.27), in an appropriate uniform sense.

THEOREM 3.5. Under the assumptions of Theorem 3.4, let us assume that $(A, B)$ satisfy one of the additional assumptions, (1.6) or (1.7). Then the function $V$ is such that, for all $y \in \mathbb{R}$ and all $t \geq 0$,

$$
\begin{gathered}
V_{t}=(1+i \alpha) V_{y y}+\mu V-(1+i \beta) V|V|^{2}+(\gamma+i \delta) V\left\langle|V|^{2}\right\rangle+\varphi(y, t), \\
V(y+1, t)=V(y, t) \exp (-i a), \\
V(y, t)=A(y+t / \varepsilon, t)+\psi(y, t),
\end{gathered}
$$

where the functions $\varphi$ and $\psi$ satisfy

$$
\begin{array}{cc}
\|\varphi(\cdot, t)\|_{\infty}, \quad\|\psi(\cdot, t)\|_{\infty} \leq K_{0} \varepsilon^{1 / 5} & \text { if } t \geq 0 \\
\|\varphi(\cdot, t)\|_{\infty}, \quad\|\psi(\cdot, t)\|_{\infty} \leq C_{0} \varepsilon^{1 / 5} & \text { if } t \geq T
\end{array}
$$

for some constants $K_{0}, T$, and $C_{0}$ such that $K_{0}$ and $T$ (respectively, $C_{0}$ ) depend only on $\|A(\cdot, 0)\|_{H^{2}(10,1)}, \beta, \gamma, \delta$, and $\mu$ (respectively, on $\beta, \gamma, \delta$, and $\mu$ ). Here the average (.), the norm $\|\cdot\|_{\infty}$ and the space $H^{2}(] 0,1[)$ are as defined in Theorem 3.4 .

Proof. The result is obtained from that in Theorem 3.4 when taking into account that if $(A, B)$ satisfy one of the additional assumptions, $(1.6)$ or $(1.7)$, then $\left\langle|V|^{2}\right\rangle=$ $\left\langle|W|^{2}\right\rangle$ for all $t \geq 0$.

Remark 3.6. According to Theorem 3.4 (respectively, Theorem 3.5), each solution of (1.1)-(1.3) (respectively, of (1.1)-(1.3), (1.6) or (1.1)-(1.3), (1.7)) satisfies approximately, in the uniform sense of the estimates (3.15) (respectively, (3.30)), the model (1.20)-(1.23) (respectively, (1.25)-(1.26)). The estimates (3.16) (respectively, (3.31)) are independent of the particular solution of (1.1)-(1.3) (respectively, of (1.1)-(1.3), (1.6) or (1.1)-(1.3), (1.7)) that is considered, but they apply only for sufficiently large time. 
3.3. Some basic properties of the approximate models. Here we give two basic properties of the approximate models, namely, that they are well posed and possess a globally attracting set.

THeOREM 3.7. If $\gamma<1$ and the functions $A_{0}$ and $B_{0}$ (respectively, $A_{0}$ ) belong to the complexified space of $C^{2+\alpha}([0,1])$, for some $\left.\alpha \in\right] 0,1[$, and satisfy (1.22) (respectively, (1.26)), then the problem (1.20)-(1.23) (respectively, (1.25)-(1.26)) possesses a tunique solution, $A, B \in$ (respectively, $A \in) C^{2+\alpha, 1+\alpha / 2}([0,1] \times[0, T])$ for all $T>0$. In addition there is a constant $C$, depending only on $\alpha, \beta, \gamma, \delta$, and $\mu$, such that, for each solution of (1.20)-(1.23) (respectively, (1.25)-(1.26)) there is a constant $T$ that satisfies, for $k=0$ and 1 ,

$$
\left.\left\|A(\cdot, t)_{C^{k}([0,1])}+\right\| B(\cdot, t) \|_{C^{k}([0,1])} \leq C \quad \text { (respectively, }\|A\|_{C^{k}([0,1])} \leq C\right)
$$

for all $t \geq T$.

Proof. The proof of the first statement is completely similar to that of Theorem 3.1, and the second statement is proven by the ideas in the proof of Lemma 3.3.

Remark 3.8. If, in addition to the assumptions in Theorem $3.7, \mu \leq 0$, then the constant $C$ may be taken arbitrarily small; this means that the trivial solutions of (1.20)-(1.23) and (1.25)-(1.26), $A \equiv B \equiv 0$ and $A \equiv 0$, are globally, asymptotically stable in this case. Therefore, if $\mu \leq 0$, then the dynamics of the approximate models are trivial. If $\gamma=1$ (respectively, $\gamma<1$ ), then both approximate models possess solutions that are unbounded as $t \rightarrow \infty$ (respectively, that blow up in a finite time), as is seen by considering spatially uniform solutions of both models.

4. Concluding remarks. We have considered the models (1.1)-(1.3), (1.1)(1.3), (1.6), and (1.1)-(1.3), (1.7) that, as it was explained in $\S 1$, are normal forms that apply at the onset of the so-called oscillatory instability in a large variety of physical problems. In the limit $\varepsilon \rightarrow 0$, we obtained (formally in $\S 2$, and rigorously in \$3) two simpler approximate models, (1.20)-(1.23) and (1.25)-(1.26). In addition, we have seen that both models are well posed, and possess a globally attracting set. Some remarks concerning these models are in order.

(A) Both aproximate models possess an inertial manifold of finite dimension. This result may be seemingly proved by extending the analysis in [18], on the standard cubic complex Ginzburg-Landau equation.

(B) The approximate model (1.20)-(1.23) possesses a two-parameter family of travelling waves of the form $V=R \exp (i \omega t+i k y+i d), W=0$, or $V=0, W=$ $R \exp (i \omega t+i k y+i d)$ for appropriate values of the real constants $R, \omega, k$, and $d$, and a four-parameter family of quasi-periodic waves of the form $V=R_{1} \exp \left(i \omega_{1} t+i k_{1} y+\right.$ $\left.i d_{1}\right), W=R_{2} \exp \left(i \omega_{2} t+i k_{2} y+i d_{2}\right)$, for appropriate values of the real constants $R_{1}$, $R_{2}, \omega_{1}, \omega_{2}, k_{1}, k_{2}, d_{1}$, and $d_{2}$ (see [19]). These wave-like solutions may lose their stability either under uniform or under nonuniform perturbations; in the second case, the solutions are said to be modulationally unstable. At the onset of the modulational instability, the model (1.20)-(1.23) may be reduced to the Kuramoto-Sivashinsky equation (see [19]) that, as is well known, exhibits chaotic behavior. Therefore, we may expect chaotic solutions of $(1.20)-(1.23)$ for appropriate values of the parameters. The same conjecture can be made in connection with the approximate model (1.25), (1.26).

(C) The particular wave-like solutions of (1.20)-(1.23) and (1.25), (1.26) mentioned in (B) are such that the moduli of $V$ and $W$ are spatially uniform. More general solutions, with spatially nonuniform modulus, are of great physical interest; 
in particular, they provide a description of the so-called blinking states of the original model (1.8), which have been detected in experiments (see [10], [11]). Both models are expected to possess such solutions. In particular, if $\alpha=\beta=\delta=0$, then it may be seen that every stationary solution of (1.25), (1.26) is such that either (i) $\theta=$ constant or (ii) $R \neq 0$ for all $y \in[0,1]$, where $R$ and $\theta$ are the modulus and the argument of $V$. The stationary solutions of type (i) may be obviously obtained in closed form, in terms of elliptic functions. The solutions of type (ii) are readily seen to be such that $d \theta / d x=c / R^{2}$ for some constant $c \neq 0$; then $R$ satisfies $R^{\prime \prime}+\mu R-R^{3}-c^{2} / R^{3}+\gamma R \int_{0}^{1} R^{2} d y=0$, and this equation is again solved in terms of elliptic functions. In addition, the evolution problem (1.25), (1.26) is gradient-like (admits the Lyapunov function $\varphi: H^{2}(] 0,1[) \rightarrow \mathbf{R}$ defined by $\varphi(V) \equiv$ $\left.\int_{0}^{1}\left(\left|V^{\prime}(y)\right|^{2}-\mu|V(y)|^{2}\right)+\int_{0}^{1}\left(|V(y)|^{4} / 2\right) d y-\gamma\left[\int_{0}^{1}|V(y)|^{2} d y\right]^{2}\right)$, and the stability of these solutions is readily analyzed. We do not include a complete study of these solutions and of their stability because it has been announced in [12]. Sufficient conditions for the existence of stable solutions of (1.25), (1.26) in the general (nonreal coefficients) case would be of great interest.

\section{REFERENCES}

[1] P. CoUllet, S. Fauve, and E. Tirapegui, Large scale instability of nonlinear standing waves, J. Physique Lett., 46 (1985), pp. L787-L791.

[2] S. FAUVE, Lange scale instabilities of cellular flows, in Instabilities and Nonequilibrium Structures, E. Tirapegui and D. Villarroel, eds., Reidel, Dordrecht, the Netherlands, 1987, pp. 63-88.

[3] P. C. Hohenberg AND M. C. CRoss, An introduction to pattern formation in non-equilibrium systems, in Lecture Notes in Phys,, Vol. 268, Springer-Verlag, Berlin, 1987, pp. 55-92.

[4] A. C. Newels, Dynamics of patterns: a survey, NATO Workshop on Propagation in Nonequilibrium Systems, held at les Houches 1989 , Springer-Verlag, Berlin.

[5] M. C. Cross, Trueling and standing waves in binary-fitid convection in finite geometries, Phys. Rev. Lett., 57 (1986), Pp. 2935-2938.

[6] __ Structure of non-linear traveling-wave states in finite geometries, Phys. Rev. A, 38 (1988), pp. 3593-3600.

[7] C. M. SuRKo AND P. KOLODNER, Oscillatory traveling-wave convection in a finite container, Phys. Lett., 58 (1987), pp. 2055-2058.

[8] E. Moses, J. Fineberg, AND V. SteinBerg, Multistability and confined travelling-wave pattertws in a convecting binary mixture, Phys. Rev. A, 35 (1987), pp. 2757-2760.

[9] R. Heinrichs, G. A hLERS, AND D. S. CANNEL, Traveling waves and spatial variation in the convection of a binary mixture, Phys. Rev. A, 35 (1987), pp. 2761-2764.

[10] J. Fineberg, E. Moses, AND V. STEINBERg, Spatially and temponally modulated traveling-wave pattern in convecting binary mixtures, Phys. Rev. Iett., 61 (1988), pp. 838-841.

[11] P. Kolodner AND C. M. Surko, Weakly non-linear travelling-wave convection, Phys. Rev. Lett., 61 (1988), pp. 842-845.

[12] E. KNOBLOCH AND J. DE LUCA, Amplitude equations of travelling wave convection, preprint, 1990.

[13] C. Alvarez-PEREIRA AND J. M. VEGA, On the pulsating instability of two-dimensional flames, European J. Appl. Math., 3 (1992), pp. 55-73.

[14] J. M. Ghidaglia, Etude d'écoulements de fluides visqueur incompressibles: comportement por les grands temps et applications aux attracteurs, Thèse de $3 \mathrm{e}$ Cycle, Université Paris Sud, Orsay, 1984.

[15] R. TEMAM, Infinite-Dimensional Dymamical Systems in Mechanics and Physics, Springer-Verlag, Berlin, 1988.

[16] D. Henfy, Geometric Theory of Semulinear Panabolic Equations, Springer-Verlag, Berlin, 1981.

[17] O. A. Ladyżenskaja, V. A. Solonnikov, AND N. N. URAl'ceva, Linear and Quagilinear Equations of Parabolic Type, American Mathematical Society, Providence, RI, 1968. 
[18] C. R. Doering, J. D. Gibbon, D. D. holm, And B. Nicolaenko, Finite Dimentionality in the Complex Ginzburg-Landau Equation, Contemporary Mathematics, 99, American Mathematical Society, Providence, RI, 1989.

[19] C. MARTEL AND J. M. VEGA, The oscillatory instability of plane wave-fronts of reaction-diffusion systems, in preparation. 